\title{
Spectral-Spatial Rotation Forest for Hyperspectral Image Classification
}

\author{
Junshi Xia, Member, IEEE, Lionel Bombrun, Yannick Berthoumieu, Member, IEEE, Christian Germain, and \\ Peijun Du, Senior Member, IEEE
}

\begin{abstract}
Rotation Forest (RoF) is a recent powerful decision tree (DT) ensemble classifier of hyperspectral images. RoF exploits random feature selection and data transformation techniques to improve both the diversity and accuracy of DT classifiers. Conventional RoF only considers data transformation on spectral information. To overcome this limitation, we propose a spectral and spatial Rotation Forest (SSRoF), to further improve the performance. In SSRoF, pixels are first smoothed by the multi-scale (MS) spatial weight mean filtering. Then, spectralspatial data transformation,which is based on a joint spectral and spatial rotation matrix, is introduced into the RoF. Finally, classification results obtained from each scale are integrated by a majority voting rule. Experimental results on two datasets indicate the competitive performance of the proposed method when compared to other state-of-the-art methods.
\end{abstract}

Index Terms-Rotation Forest, spectral-spatial transformation, classification ensemble, hyperspectral images

\section{INTRODUCTION}

Hyperspectral image (HSI) records the reflectance in the range of visible, NIR and SWIR to monitor the earth's surface to make them widely used in mineral identification and agricultural mapping [1], [2]. Classifying pixels given in the scene are often included in these applications. However, due to the high dimensionality [3], the spectral/spatial redundancy [4], [5], the spectral variability [6] and hyperspectral absorption band mapping [7], [8] of hyperspectral data, as well as the limited number of training samples, HSI classification is a challenging task.

In recent years, many kinds of research were devoted to design pixel-wise classifiers, which only processes each pixel independently and adopt its spectral information as the input to the classifier. Kernel methods, such as the support vector

Manuscript received; revised. This study has been carried out with financial support from the French State, managed by the French National Research Agency (ANR) in the frame of the "Investments for the future" Programme IdEx Bordeaux-CPU (ANR-10-IDEX-03-02).

J. Xia is with the Research Center for Advanced Science and Technology, The University of Tokyo, Tokyo, Japan and with Université de Bordeaux, Bordeaux INP, and CNRS, Laboratoire IMS, UMR 5218, F-33405 Talence, France (e-mail: xiajunshi@gmail.com).

L. Bombrun, Y. Berthoumieu and C. Germain are with Université de Bordeaux, Bordeaux INP, and CNRS, Laboratoire IMS, UMR 5218, F-33405 Talence, France (e-mail: \{lionel.bombrun,yannick.berthoumieu,christian.germain\}@ims-bordeaux.fr).

P. Du is with the Key Laboratory for Satellite Mapping Technology and Applications of National Administration of Surveying, Mapping and Geoinformation of China, Jiangsu Provincial Key Laboratory of Geographic Information Science and Technology, Jiangsu Center for Collaborative Innovation in Geographical Information Resource Development and Application, and Collaborative Innovation Center of South China Sea Studies, Nanjing University, 210023 Nanjing, China (dupjrs@gmail.com). machines (SVMs), have proven to be a robust classifier of hyperspectral data, due to their ability in addressing both the small-size-sample problems and the curse of dimensionality [9], [10].

Multiple classifier systems (MCSs) or classifier ensemble, which produce the final output based on the decisions made by a set of individual classifiers according to certain rules, have been a hot topic in the hyperspectral remote sensing community [5], [11]. MCSs is very effective due to complementary and diverse information provided by the member classifiers, thus enhancing the classification performance [12]. Random Forest (RF) [13] is the most popular ensemble classifier in the hyperspectral remote sensing community. In RF, each decision tree is trained on a bootstrapped training set, and the randomly selected features are used to split a node in a decision tree. Rotation forest (RoF) is one of the current state-of-the-art decision tree ensemble classifier [14]. In contrast to RF, RoF splits the features into several disjoint subsets and uses the bootstrap aggregation (Bagging) technique on each subgroup to select the bootstrapped training set for applying data transformation. Then, a new training set for the decision tree (DT) is formed by concatenating the linear extracted features contained in each subset. Thus, RoF enhances both accuracy and diversity within the ensemble and achieves better performance than Bagging, Boosting, and RF [14]. Our previous studies [15], [16] supported the superiority of RoF. RoF is competitive with the SVMs, and needs shorter computational time and fewer parameters to tune. Studies on the use of RoF and its variants dealing with hyperspectral classification problems have been recently published [15]-[19]. Furthermore, RoF has proven to be effective not only for hyperspectral data analysis but also for very high spatial resolution optical and SAR images [20], [21]. Although RoF obtains remarkable performance, data transformation in RoF is performed only by measuring the similarity between the samples using spectral information (i.e., Euclidean distance) [22]. However, this is insufficient to reveal the intrinsic relationship between different samples [23]. To improve the performance, we introduce the spatial information to compute the sample similarity [23].

Pixel-wise classifiers do not exploit the relationship among the neighboring pixels [6], [24]. However, these adjacent pixels sometimes show similar spectral characteristics. It is necessary to develop a spectral-spatial classifier which takes into account both the spectral and spatial information. For instance, Chen $e t$ al. developed a spectral-spatial classifier based on kernel extreme learning machine [25]. Chen et al. extended deep belief network into the spectral-spatial framework [26]. Due 
to the excellent performance of RoF, a new spectral-spatial approach was developed in [16] integrating RoF with local feature extraction. The spatial information was represented by Markov random fields (MRF)-based multilevel logistic (MLL) prior. We refer this method to RoF-MRF. Another popular strategy to exploit the spatial information is the filteredbased approaches $[27]-[30]$. The motivation is due to the fact that the filtering methods are effective at removing noise in the regions, but adversely affect edges. In this case, the performance is significantly improved. Kang et al. [28], [29] combined a probabilistic SVM with an edge-preserving filter (EPF) and proposed a spatial feature extraction method with image fusion and recursive filtering techniques (IFRF). The main challenge of the above filtering method is to focus on one scale, which may not work well for a more complex area because landscapes typically consist of different types of land covers that vary in size (e.g., trees and buildings). To mitigate this problem, we adopt the multi-scale filtering methods.

In this paper, we incorporate the spatial information into the process of RoF, and thus, propose a spectral and spatial RoF (SSRoF) method. In SSRoF, we exploited the information into two different ways: spatial filtering and spectral-spatial data transformation into the RoF. More specifically, a multi-scale weighted mean filtering (WMF) is used to preprocess the HSI because the WMF is both efficient and fast. Then, we introduce the spectral-spatial data transformation that combines local Fisher discriminant analysis (LFDA) [22] and spatial-domain data transformation [23], into RoF. Finally, we use a majority vote rule to combine the results generated by each scale.

The proposed SSRoF extends the traditional RoF by using both spectral and spatial information with the following characteristics:

- The spatial filtering increases the adjacent pixel consistency. The WMF method brings some dependency between neighboring pixels and removes the noise and preserves the primary information of the images in an efficient way.

- By introducing spectral and spatial data transformation into RoF, we can increase the accuracy of member DT classifiers and diversity within the ensemble, to further improve the performance of RoF.

- The multi-scale method can describe different spatial structures of HSIs. The rich complementary information in the multi-scale filtered datasets thus helps to improve the performance of the subsequent classification results.

The remainder of this paper is organized as follows. Section II presents the proposed method. Section III presents the experimental results and analysis. Conclusions and perspectives are provided in Section IV

\section{PROPOSED METHOD}

The flowchart of the proposed method is depicted in Fig. 1 It consists of the following steps:

- Spatial WMF with $N$ scales are used to preprocess the HSI pixels.

- SSRoF is used to classify the multi-scale filtered HSIs.
- The final result is generated by combining the classification maps of each scale by a majority vote rule.

We detail in the next subsection each of these steps.

\section{A. Spatial WMF}

A spatial WMF [31] is used to preprocess the HSI pixels with the aim of reducing the noise and smoothing the homogeneous regions.

Let us denote the coordinates of sample $\mathbf{x}_{i}$ as $\left(\alpha_{i}, \beta_{i}\right)$, the local pixel neighborhood with the center pixel $\mathbf{x}_{i}$ is defined as:

$\mathcal{N}=\left\{\mathbf{x}=(\alpha, \beta) \mid \alpha \in\left[\alpha_{i}-a, \alpha_{i}+a\right], \beta \in\left[\beta_{i}-a, \beta_{i}+a\right]\right\}$

where $\omega=2 a+1$ is the scale that represents the width of the neighborhood window.

The spatial WMF of $\mathbf{x}_{i}$ is calculated by

$$
\tilde{\mathbf{x}}=\frac{\sum_{\mathbf{x}_{j} \in \mathcal{N}\left(\mathbf{x}_{i}\right)} \mu_{j} \mathbf{x}_{j}}{\sum_{\mathbf{x}_{j} \in \mathcal{N}\left(\mathbf{x}_{i}\right)} \mu_{j}}=\frac{\mathbf{x}_{i}+\sum_{k=1}^{\omega^{2}-1} \mu_{k} \mathbf{x}_{i k}}{1+\sum_{k=1}^{\omega^{2}-1} \mu_{k}}
$$

where, $\mathbf{x}_{i}, \mathbf{x}_{i 1}, \ldots, \mathbf{x}_{i s}$ are the pixels in the neighborhood of $\mathbf{x}_{i} . s=\omega^{2}-1$ is the number of neighbors. $\mu_{k}=$ $\exp \left\{-\tau\left\|\mathbf{x}_{i}-\mathbf{x}_{i k}\right\|^{2}\right\}$ is the weight to measure spectral distances of the neighboring pixels to $\mathbf{x}_{i} . \tau$ is the filtering degree that is empirically set to be 0.3 . Furthermore, we adapt a multi-scale approach in order to capture both small and large structures in HSI. In this case, we can improve the performance of the subsequent SSRoF classifier by integrating the rich complementary information in the multi-scale filter datasets.

\section{B. Spectral-spatial Rotation Forest (SSRoF)}

In this part, we first introduce the spectral-spatial data transformation and then the main steps of spectral-spatial rotation forest.

Let us denote $\{\mathbf{X}, \mathbf{Y}\}=\left\{\left(\mathbf{x}_{1}, y_{1}\right), \ldots,\left(\mathbf{x}_{n}, y_{n}\right)\right\}$ as the training samples, where $\mathbf{x}_{i} \in \mathbb{R}^{D}$ is a pixel and $y_{i}$ is a scalar with classes of interest $\mathcal{C}=\{1, \ldots, C\}$, where $C$ is the total number of classes.

For data transformation, we often assume that there exists a mapping function $f: \mathbb{R}^{D} \rightarrow \mathbb{R}^{d}, d \leq D$, which can transform each data point $\mathbf{x}_{i}$ to $\mathbf{z}_{i}=f\left(\mathbf{x}_{i}\right)$. This mapping is represented by a $D \times d$ matrix $\mathbf{V}$ :

$$
\mathbf{z}_{i}=f\left(\mathbf{x}_{i}\right)=\mathbf{V}^{\top} \mathbf{x}_{i}
$$

The projection matrix $\mathbf{V}=\left(\mathbf{v}_{1}, \mathbf{v}_{2}, \ldots \mathbf{v}_{d}\right)$ is obtained as the $d$ eigenvectors corresponding to the $d$ largest eigenvalues $\left\{\lambda_{1}, \lambda_{2}, \ldots, \lambda_{d}\right\}$, by solving the following generalized eigenvalue decomposition equation:

$$
\mathbf{S}_{1} \mathbf{v}=\lambda \mathbf{S}_{2} \mathbf{v}
$$

where, $\mathbf{S}_{1}$ and $\mathbf{S}_{2}$ are matrices which depend on the considered data transformation approach. 


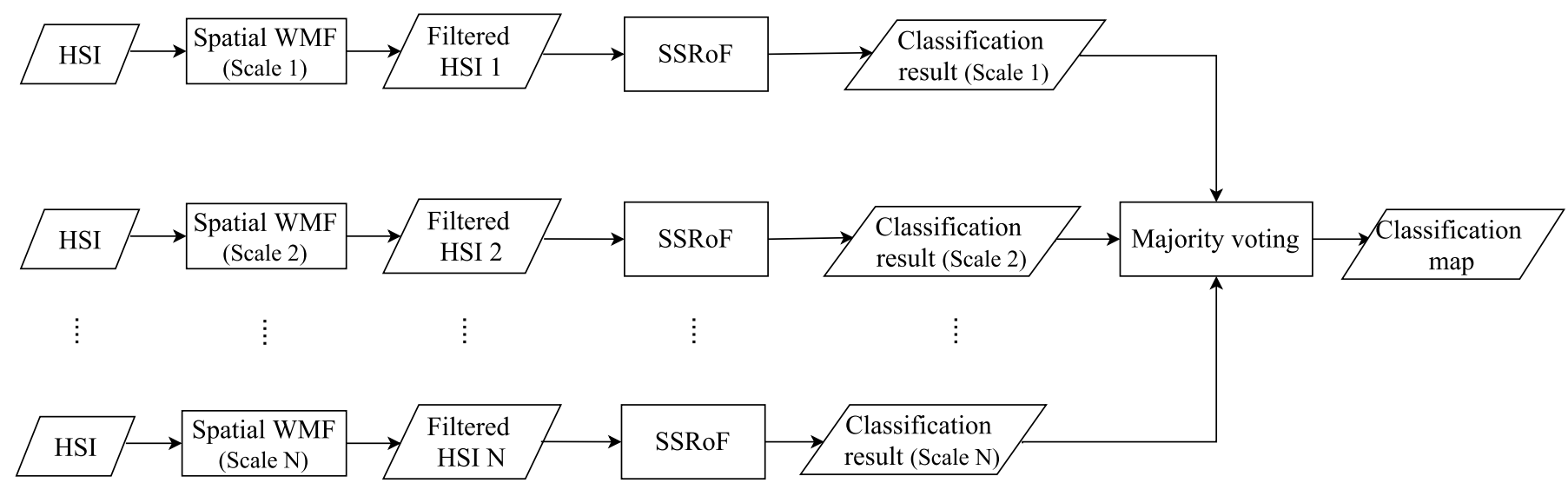

Fig. 1. Flowchart of the proposed method.

1) Spectral-domain data transformation: LFDA is used as the spectral-domain data transformation. LFDA effectively combines the ideas of Fisher discriminant analysis (FDA) and locality-preserving projection (LPP). Hence, LFDA maximizes the between-class separability and preserves the within-class local structure [22]. Practically, LFDA is obtained by solving the following eigenvalue decomposition equation:

$$
\mathbf{S}^{l b} \mathbf{v}=\lambda \mathbf{S}^{l w} \mathbf{v}
$$

where, $\mathbf{S}^{l b}$ and $\mathbf{S}^{l w}$ denote respectively the local between-class and the within-class scatter matrix, which is given by

$$
\begin{aligned}
\mathbf{S}^{l b} & =\frac{1}{2} \sum_{i, j=1}^{n} \omega_{i, j}^{l b}\left(\mathbf{x}_{i}-\mathbf{x}_{j}\right)\left(\mathbf{x}_{i}-\mathbf{x}_{j}\right)^{\top} \\
\mathbf{S}^{l w} & =\frac{1}{2} \sum_{i, j=1}^{n} \omega_{i, j}^{l w}\left(\mathbf{x}_{i}-\mathbf{x}_{j}\right)\left(\mathbf{x}_{i}-\mathbf{x}_{j}\right)^{\top}
\end{aligned}
$$

where $\omega^{l b}$ and $\omega^{l w}$ are $n \times n$ matrices defined as

$$
\begin{gathered}
\omega_{i, j}^{l b}=\left\{\begin{aligned}
A_{i, j}\left(\frac{1}{n}-\frac{1}{n_{y_{i}}}\right) & \text { if } y_{i}=y_{j} \\
\frac{1}{n} & \text { otherwise }
\end{aligned}\right. \\
\omega_{i, j}^{l w}=\left\{\begin{aligned}
\frac{A_{i, j}}{n_{y_{i}}} & \text { if } y_{i}=y_{j} \\
\frac{1}{n} & \text { otherwise }
\end{aligned}\right.
\end{gathered}
$$

where $n_{y_{i}}$ is the number of labeled samples in class $y_{i} \in \mathcal{C}$ and $A_{i, j}$ is defined as the affinity between $\mathbf{x}_{i}$ and $\mathbf{x}_{j}$ given by

$$
A_{i, j}=\exp \left(-\frac{\left\|\mathbf{x}_{i}-\mathbf{x}_{j}\right\|}{\sigma_{i} \sigma_{j}}\right)
$$

where, $\sigma_{i}=\left\|\mathbf{x}_{i}-\mathbf{x}_{i}^{k}\right\|$ denotes the local scaling of $\mathbf{x}_{i}$ and $\mathbf{x}_{i}^{k}$ is the $k$-th nearest neighbor of $\mathbf{x}_{i}$ in the spectral domain of training set ( $k$ is set to 3 ).

2) Spatial-domain data transformation: In a spatial local homogeneous region, neighboring pixels belongs to the same materials and are within the same class [23]. Under this situation, the spatial-domain data transformation proposed in [23] are adopted. Compared to the spectral-domain data transformation, it uses spatial information to learn the projections. More specifically, spatial-domain data transformation is obtained by solving the following eigenvalue decomposition equation:

$$
\mathbf{S v}=\lambda \mathbf{H v}
$$

where $\mathbf{H}$ and $\mathbf{S}$ are defined as the local pixel neighborhood preserving matrix and the total scatter matrix, respectively.

$\mathbf{H}$ is defined as

$$
\mathbf{H}=\sum_{i=1}^{n} \sum_{k=1}^{s} \frac{\mu_{k}}{\sum_{j=1}^{s} \mu_{j}}\left(\mathbf{x}_{i}-\mathbf{x}_{i k}\right)\left(\mathbf{x}_{i}-\mathbf{x}_{i k}\right)^{\top}
$$

where $\mathbf{x}_{i k}$ is the $k$-th neighbor of $\mathbf{x}_{i}$ in its spatial neighbors in $\mathcal{N}\left(\mathbf{x}_{i}\right) . s$ is the number of spatial neighbors.

$\mathbf{S}$ is given by:

$$
\mathbf{S}=\sum_{i=1}^{n}\left(\mathbf{x}_{i}-\mathcal{M}\right)\left(\mathbf{x}_{i}-\mathcal{M}\right)^{\top}
$$

where $\mathcal{M}$ is the mean of training samples $\mathbf{X}$.

3) Spectral-spatial data transformation: In order to tackle the spectral and spatial information into data transformation process, we combine LFDA and spatial-domain data transformation to obtain a spectral-spatial data transformation that preserves not only the spectral-domain local Euclidean neighborhood class information but also the spatial-domain local pixel neighborhood structures [23].

Thus, the spectral-spatial local neighborhood preserving scatter matrix and a modified total scatter matrix are defined as $\phi \mathbf{S}^{l w}+(1-\phi) \mathbf{H}$ and $\phi \mathbf{S}^{l b}+(1-\phi) \mathbf{S}$, respectively [23].

The projection in spectral-spatial data transformation is achieved by making the trade-off between the eigendecomposition in spectral (Eq. (5p) and spatial (Eq. (11p) data transformation [23]:

$$
\left(\phi \mathbf{S}^{l b}+(1-\phi) \mathbf{S}\right) \mathbf{v}=\lambda\left(\phi \mathbf{S}^{l w}+(1-\phi) \mathbf{H}\right) \mathbf{v}
$$

where $\phi$ is the control parameter of spectral and spatial information. When $\phi=1$ (resp. $\phi=0$ ), the spectral-spatial data transformation is reduced to spectral case. When $\phi=0$, spectral-spatial data transformation is reduced to the spectral (resp. spatial) one.

4) Main steps of SSRoF: Spectral-spatial Rotation Forest (SSRoF) is a variant of RoF, which uses spectral-spatial data transformation. The main training and prediction steps are presented in Algorithm 1 


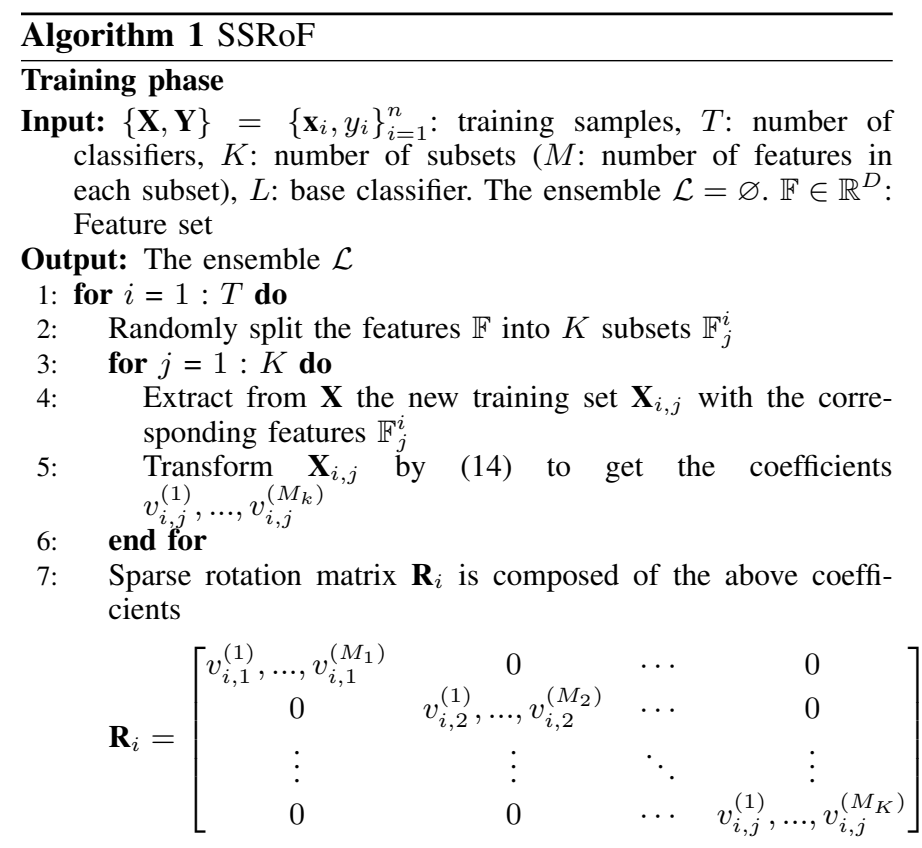

\section{EXPERIMENTS AND ANALYSIS}

In this section, the proposed method is investigated on the two benchmark hyperspectral datasets.

\section{A. Hyperspectral datasets}

1) Indian Pines: This image was obtained by the Airborne Visible/Infrared Imaging Spectrometer (AVIRIS) sensor over the Indian Pines in Northwestern Indiana, USA. It contains 220 spectral bands in the wavelength range from 0.4 to 2.5 $\mu m$ (spectral resolution:10 $\mathrm{nm}$ ) and is composed of $145 \times 145$ pixels (ground sampling distance (GSD): $20 \mathrm{~m}$ ). The reference data with 16 classes of interest consist of 10336 pixels. Threeband color composite image and the ground truth of AVIRIS hyperspectral data are shown in Fig. 2 .

2) University of Pavia: This image is of an urban area operated by a Reflective Optics Spectrographic Imaging System (ROSIS)-03 optical airborne sensor. It consists of $610 \times 340$ pixels with a GSD of $1.3 \mathrm{~m}$. The 12 noisy bands have been removed, and the remaining 103 bands have been used in the classification. The reference data with nine classes of interest is composed of 42776 pixels. Three-band color composite image and the ground truth are presented in Fig. 3 .

$$
\text { of votes. } y_{*}=\underset{i \in\{1,2, \ldots, C\}}{\operatorname{argmax}} \sum_{j: L_{j}\left(\mathbf{x}_{*}^{\top} \mathbf{R}_{i}^{a}\right)=i}^{T} 1
$$

In the training phase, the ensemble is learned as follows:

- the first step consisting in splitting the feature space of training set $\mathbb{F}$ into $K$ disjoint subsets, and each contains $M$ features.

- spectral-spatial data transformation is performed on each subset to obtain the projection matrix. It should be noted that the numbers of input and output features are both set to be $M$.

- a sparse rotation matrix $\mathbf{R}_{i}$ is generated by the projection matrix in each subset.

- a rotated training set is generated by rotating the original training set with a rearranged sparse matrix $\mathbf{R}_{i}^{a}$.

- an individual DT classifier is trained on this rotated training set.

- the ensemble is composed of several DT classifiers by repeating the above steps $T$ times.

In the prediction phase, a new sample $\mathbf{x}_{*}$ is rotated by $\mathbf{R}_{i}^{a}$. Then, the transformed set, i.e., $\mathbf{x}_{*}^{\top} \mathbf{R}_{i}^{a}$, is classified by the ensemble of several decision trees and the class with the maximum number of votes is chosen as the final class.

As explained before, SSRoF extends both SpeRoF and SpaRoF. These latter are respectively obtained by setting $\phi=1$ and $\phi=0$ in 14 .

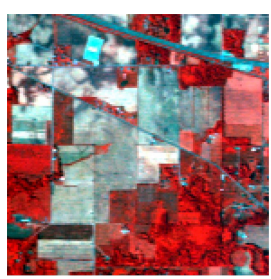

(a)

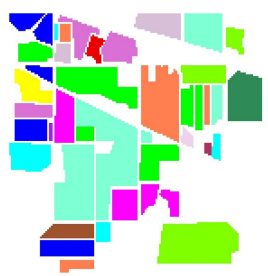

(b)

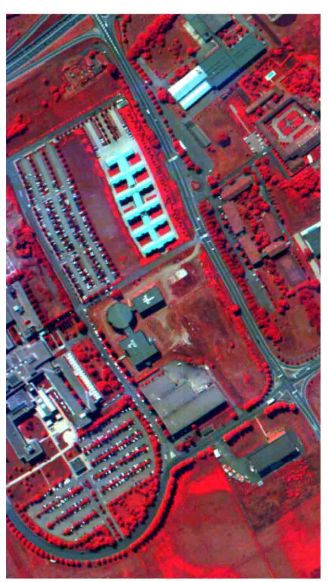

(a)

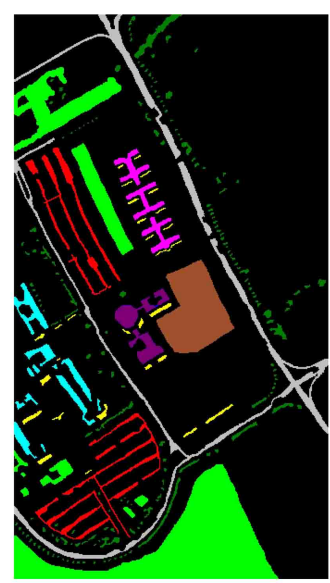

(b)

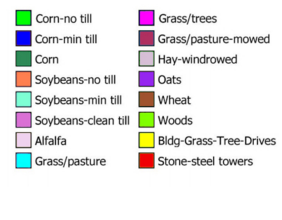

Fig. 2. (a) Three-band color composite of AVIRIS image. (b) Ground truth.
Fig. 3. (a) Three-band color composite of ROSIS image. (b) Ground truth.

\section{B. Compared methods}

The following methods are used for comparisons:

- MLRsubMLL [32], a Bayesian approach that contains two components: 1) the posterior probability is achieved 
TABLE I

OVERALl CLASSIFICATION RESUlts (\%) OBTAINED FOR THE INDIAN PINES AVIRIS IMAGE USING DIFFERENT NUMBERS OF TRAINING SAMPLES PER CLASS.

\begin{tabular}{|c|c|c|c|c|c|c|c|c|c|}
\hline Number of training samples per class & 10 & 15 & 20 & 25 & 30 & 35 & 40 & 45 & 50 \\
\hline MLRsubMLL & 61.75 & 63.89 & 66.75 & 67.21 & 68.37 & 71.02 & 74.86 & 76.17 & 79.34 \\
\hline RoF-MRF & 73.43 & 77.86 & 81.74 & 83.28 & 85.72 & 86.37 & 87.68 & 88.67 & 91.03 \\
\hline RS-EMAPs & 82.14 & 85.21 & 87.64 & 88.62 & 90.86 & 92.16 & 92.45 & 93.64 & 93.75 \\
\hline SVM-CK & 80.74 & 82.68 & 84.94 & 86.21 & 87.52 & 89.16 & 90.15 & 91.25 & 91.98 \\
\hline EPF & 61.01 & 65.89 & 72.34 & 76.45 & 79.87 & 81.42 & 83.14 & 85.01 & 86.21 \\
\hline IFRF & 78.21 & 83.45 & 87.24 & 88.64 & 90.42 & 90.86 & 91.69 & 92.97 & 93.48 \\
\hline RF & 46.49 & 54.47 & 57.03 & 60.19 & 60.67 & 61.89 & 63.38 & 64.62 & 65.19 \\
\hline SpeRoF & 57.73 & 65.33 & 66.74 & 72.00 & 73.17 & 73.57 & 75.87 & 76.29 & 77.74 \\
\hline SpaRoF & 58.98 & 66.21 & 68.20 & 72.48 & 73.65 & 73.99 & 76.71 & 77.01 & 77.57 \\
\hline SSRoF & 60.05 & 66.97 & 68.53 & 72.69 & 74.48 & 74.58 & 76.91 & 77.31 & 78.12 \\
\hline SpeRoF $_{o p t}$ & 78.51 & 80.75 & 84.38 & 86.86 & 88.45 & 90.23 & 91.98 & 92.50 & 92.97 \\
\hline $\mathrm{SpaRoF}_{\text {opt }}$ & 78.52 & 81.17 & 85.05 & 87.02 & 88.55 & 90.36 & 92.06 & 92.24 & 92.53 \\
\hline $\mathrm{SSRoF}_{o p t}$ & 79.67 & 82.69 & 85.32 & 87.56 & 88.98 & 91.58 & 92.12 & 93.02 & 93.14 \\
\hline$\overline{\text { SpeRoF }_{M S}}$ & 80.71 & 84.04 & 85.91 & $\overline{87.84}$ & 89.32 & 91.89 & 92.99 & 93.98 & $\overline{994.07}$ \\
\hline SpaRoF $_{M S}$ & 81.27 & 84.32 & 86.36 & 88.35 & 89.45 & 92.04 & 93.30 & 94.36 & 94.93 \\
\hline $\mathrm{SSRoF}_{M S}$ & 82.55 & 85.24 & 87.45 & 88.67 & 90.21 & 92.53 & 94.40 & 94.39 & 95.12 \\
\hline
\end{tabular}

TABLE II

OVERALL CLASSIFICATION RESULTS (\%) OBTAINED FOR THE UNIVERSITY OF PAVIA ROSIS IMAGE USING DIFFERENT NUMBERS OF TRAINING SAMPLES PER CLASS.

\begin{tabular}{|c|c|c|c|c|c|c|c|c|c|}
\hline Number of training samples per class & 10 & 15 & 20 & 25 & 30 & 35 & 40 & 45 & 50 \\
\hline MLRsubMLL & 75.24 & 78.01 & 81.87 & 83.75 & 86.42 & 86.92 & 87.01 & 87.34 & 88.13 \\
\hline RoF-MRF & 78.21 & 82.15 & 85.78 & 86.21 & 88.70 & 88.64 & 91.52 & 91.99 & 92.95 \\
\hline RS-EMAPs & 85.24 & 87.64 & 89.75 & 90.75 & 92.75 & 93.04 & 93.45 & 94.02 & 94.56 \\
\hline SVM-CK & 82.74 & 85.05 & 87.24 & 88.55 & 90.18 & 91.61 & 91.73 & 92.82 & 93.02 \\
\hline EPF & 75.58 & 79.05 & 85.24 & 86.34 & 88.52 & 89.64 & 91.54 & 92.07 & 92.86 \\
\hline IFRF & 74.84 & 82.21 & 85.96 & 87.67 & 88.24 & 89.56 & 91.78 & 92.86 & 92.86 \\
\hline RF & 59.27 & 59.89 & 66.41 & 67.94 & 68.90 & 69.15 & 72.73 & 73.37 & 73.95 \\
\hline SpeRoF & 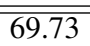 & $\overline{772.71}$ & $\overline{777.05}$ & $\overline{788.33}$ & $\overline{82.36}$ & 82.12 & 82.15 & 833.15 & $\overline{85.42}$ \\
\hline SpaRoF & 70.30 & 73.87 & 77.78 & 80.12 & 82.58 & 82.33 & 82.96 & 83.51 & 85.38 \\
\hline SSRoF & 72.05 & 74.53 & 78.66 & 80.53 & 82.73 & 83.34 & 83.68 & 84.03 & 86.04 \\
\hline$\overline{\text { SpeRoF }_{\text {opt }}}$ & 81.18 & 83.87 & 86.36 & 89.39 & 91.18 & 91.70 & 92.66 & 93.47 & $\overline{94.08}$ \\
\hline SpaRoF $_{\text {opt }}$ & 80.42 & 83.95 & 87.76 & 90.87 & 91.40 & 92.44 & 92.97 & 93.82 & 94.19 \\
\hline $\mathrm{SSRoF}_{o p t}$ & 82.51 & 84.04 & 88.22 & 90.76 & 92.02 & 92.69 & 93.07 & 94.90 & 95.08 \\
\hline SpeRoF $_{M S}$ & 82.96 & 86.78 & 88.14 & 91.01 & 93.29 & 94.12 & 94.44 & 96.21 & 97.05 \\
\hline SpaRoF $_{M S}$ & 82.73 & 87.62 & 89.86 & 91.24 & 93.78 & 94.45 & 95.66 & 97.08 & 97.10 \\
\hline $\mathrm{SSRoF}_{M S}$ & 86.80 & $\mathbf{8 8 . 3 7}$ & 90.33 & 91.53 & 93.93 & 94.68 & 95.98 & 97.21 & 97.79 \\
\hline
\end{tabular}

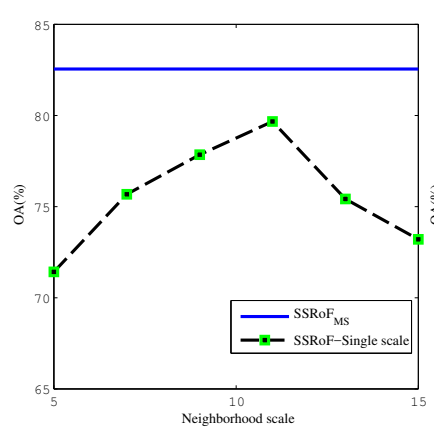

(a)

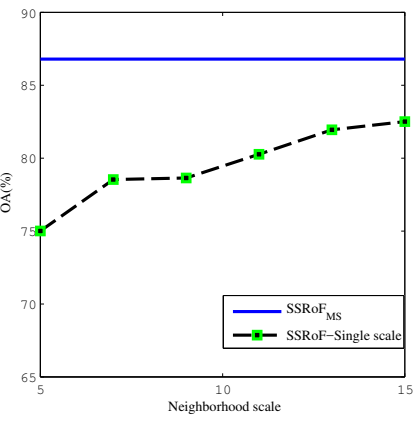

(b)
Fig. 4. Influence of the neighborhood scale on the proposed method (10 samples per class). (a) Indina Pines AVIRIS. (b) University of Pavia ROSIS.

by a subspace multinomial logistic regression (MLRsub) classifier and 2) segmentation, which refers to an image of class labels from a posterior distribution built on the aforementioned classifier and on an MLL prior.

- RoF-MRF [16], integration of RoF with local feature extraction and spatial information is represented by MRFMLL prior.

- RS-EMAPs [19], random subspace (RS) ensemble classifier with extended morphological attribute profiles (EMAPs).

- SVM-CK [33], composite kernels of SVMs for integrating spectral information and extended morphological attribute profiles (EMAPs).

- EPF [28]: a spatial regularization method for a probabilistic SVM by using an EPF.

- IFRF [29]: the extraction of spatial features with image fusion and recursive filtering (IFRF).

The parameter settings of the compared algorithms follow these of the original papers [16], [28], [29], [32]. For the 
TABLE III

INDIAN PINES AVIRIS: $Z$ VALUES OF MCNEMAR'S TEST TO VALIDATE WHETHER THE DIFFERENCE BETWEEN CLASSIFICATION ACCRUES OF THE PROPOSED METHODS IS SIGNIFICANTLY DIFFERENT FROM OTHER METHODS.

\begin{tabular}{|c|c|c|c|c|c|c|c|c|c|}
\hline Samples per class & 10 & 15 & 20 & 25 & 30 & 35 & 40 & 45 & 50 \\
\hline $\mathrm{SSRoF}_{M S}$ vs. SSRoF $\mathrm{F}_{o p t}$ & 7.62 & 6.92 & 6.71 & 2.01 & 4.75 & 2.15 & 6.18 & 2.74 & 7.15 \\
\hline SSRoF $_{M S}$ vs. SSRoF & 29.56 & 28.13 & 27.37 & 26.11 & 26.82 & 25.18 & 27.03 & 26.78 & 26.31 \\
\hline $\operatorname{SSRoF}_{M S}$ vs. MLRsubMLL & 27.81 & 30.45 & 29.88 & 32.13 & 33.42 & 28.28 & 29.32 & 27.91 & 23.14 \\
\hline $\mathrm{SSRoF}_{M S}$ vs. RoF-MRF & 19.81 & 15.62 & 12.46 & 11.74 & 10.43 & 10.39 & 10.67 & 11.42 & 12.75 \\
\hline SSRoF $_{M S}$ vs. RS-EMAPs & 1.14 & 0.72 & -0.58 & 0.94 & -0.48 & 0.78 & 3.14 & 2.06 & 3.78 \\
\hline SSRoF $_{M S}$ vs. SVM-CK & 7.81 & 6.94 & 6.47 & 6.82 & 6.12 & 7.01 & 7.23 & 7.84 & 8.45 \\
\hline SSRoF $_{M S}$ vs. EPF & 28.15 & 28.62 & 20.13 & 21.63 & 18.82 & 17.48 & 15.47 & 17.18 & 18.41 \\
\hline $\mathrm{SSRoF}_{M S}$ vs. IFRF & 13.25 & 5.92 & 1.99 & 1.97 & -0.45 & 3.78 & 5.26 & 4.26 & 4.01 \\
\hline
\end{tabular}

TABLE IV

UNIVERSITY OF PAVIA ROSIS: $Z$ VALUES OF MCNEMAR'S TEST TO VALIDATE WHETHER THE DIFFERENCE BETWEEN CLASSIFICATION ACCRUES OF THE PROPOSED METHODS IS SIGNIFICANTLY DIFFERENT FROM OTHER METHODS.

\begin{tabular}{c|c|c|c|c|c|c|c|c|c}
\hline \hline Samples per class & 10 & 15 & 20 & 25 & 30 & 35 & 40 & 45 & 50 \\
\hline SSRoF $_{M S}$ vs. SSRoF \\
Spt & 25.34 & 26.78 & 21.46 & 15.48 & 11.42 & 14.67 & 16.42 & 15.38 & 18.03 \\
SSRoF $_{M S}$ vs. SSRoF & 50.28 & 49.26 & 49.13 & 48.72 & 47.24 & 45.44 & 46.82 & 44.80 & 46.21 \\
SSRoF $_{M S}$ vs. MLRsubMLL & 33.81 & 32.24 & 31.62 & 32.74 & 31.60 & 30.48 & 29.53 & 28.14 & 30.14 \\
SSRoF $_{M S}$ vs. RoF-MRF & 25.47 & 26.98 & 24.51 & 24.80 & 27.17 & 27.63 & 28.92 & 24.45 & 20.34 \\
SSRoF $_{M S}$ vs. RS-EMAPs & 3.19 & 2.63 & 2.08 & 2.76 & 3.20 & 3.46 & 3.98 & $4 / 64$ & 5.96 \\
SSRoF $_{M S}$ vs. SVM-CK & 25.94 & 21.62 & 20.74 & 20.15 & 19.63 & 19.74 & 18.47 & 18.10 & 18.63 \\
SSRoF $_{M S}$ vs. EPF & 33.29 & 31.28 & 28.46 & 27.16 & 20.12 & 18.64 & 17.43 & 16.81 & 13.52 \\
SSRoF $_{M S}$ vs. IFRF & 34.58 & 30.01 & 28.64 & 27.52 & 24.63 & 20.06 & 18.04 & 16.74 & 12.17 \\
\hline \hline
\end{tabular}

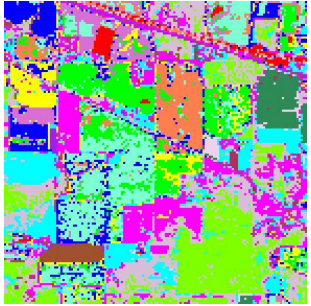

(a)

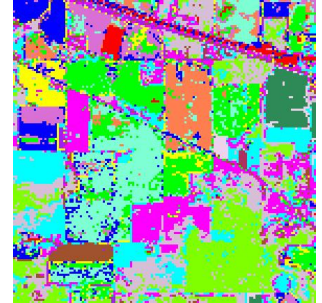

(b)

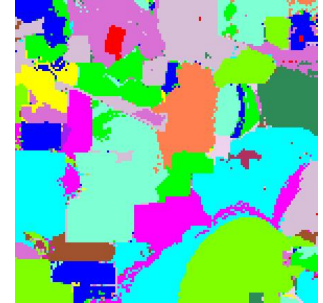

(c)

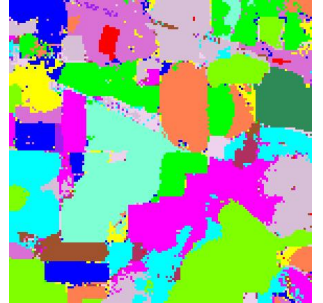

(d)

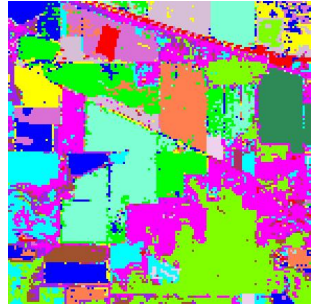

(e)

Fig. 5. Classification maps of Indina Pines AVIRIS image (20 samples per class). (a) SpeRoF, OA $=70.11 \%$. (b) $\mathrm{SSRoF}, \mathrm{OA}=72.01 \%$. (c) $\mathrm{SSRoF}$ opt , OA $=85.92 \%$. (d) $\mathrm{SSRoF}_{M S}, \mathrm{OA}=88.75 \%$. (e) RS-EMAPs, $\mathrm{OA}=87.35 \%$.

TABLE V

COMPARISON BETWEEN SSROF AND SPEROF (SPAROF). FOR EACH METHOD, "OA (\%)", "AOA (\%)", AND DIVERSITIES ARE REPORTED. 10 SAMPLES PER CLASS ARE USED AS THE TRAINING SET.

\begin{tabular}{c|ccc|ccc}
\hline & \multicolumn{3}{c}{ Indian Pines } & \multicolumn{3}{c}{ University of Pavia } \\
\hline & SpeRoF & SpaRoF & SSRoF & SpeRoF & SpaRoF & SSRoF \\
\hline OA (\%) & 57.73 & 58.98 & $\mathbf{6 0 . 0 5}$ & 69.73 & 70.30 & $\mathbf{7 2 . 0 5}$ \\
AOA (\%) & 44.68 & 44.79 & $\mathbf{4 5 . 8 7}$ & 57.41 & 57.62 & $\mathbf{5 8 . 3 4}$ \\
CFD & 46.89 & 47.32 & $\mathbf{4 7 . 8 7}$ & 55.87 & 56.82 & $\mathbf{5 9 . 0 2}$ \\
\hline
\end{tabular}

SVMs in SVM-CK, EPF, and IFRF, they are selected by a five-fold cross-validation procedure.

In addition to the aforementioned five algorithms, the methods only considering the spectral information or spatial information in single scale are added to compare to the proposed method: $\mathrm{SSRoF}_{M S}$.

- RF: RF with spectral information.

- SpeRoF: RoF only considering the spectral-based data transformation (LFDA).

- SpaRoF: RoF only considering the spatial-based data transformation.
- SSRoF: RoF considering the spectral-spatial data transformation.

- $\mathrm{SpReF}_{\text {opt }}$ or $\mathrm{SpaRoF}_{\text {opt }}$ or $\mathrm{SSRoF}_{o p t}$ : SpeRoF or SpaRoF or SSRoF with the single optimized scale, which obtains the highest accuracy.

- SpeRoF $\mathrm{R}_{M S}$ or $\mathrm{SpaRoF}_{M S}$ : SpeRoF or SpaRoF with the multi-scale features obtained after the WMF filter.

\section{Results}

In the first experiment, we present the overall accuracies (OAs) of different methods with the various numbers of training samples on Indian Pines AVIRIS and University of Pavia ROSIS image in Tables II and II] respectively. The Number of classifiers $(T)$ and the control parameter $\phi$ are set to be 20 and 0.5 , respectively. Number of features in a subset $(M)$ are respectively set to 110 and 10 on AVIRIS and ROSIS image. Six different scales, i.e., $5 \times 5, \ldots, 15 \times 15$ are considered. It can be seen that SSRoF outperforms SpeRoF and $\mathrm{SpaRoF}$ because we introduce both spectral and spatial information into the data transformation techniques. When the WMF filters are incorporated into the Rotation Forest, 


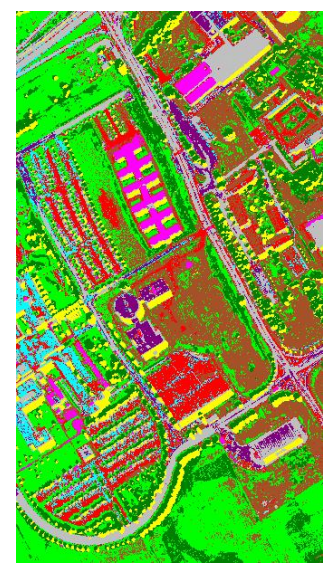

(a)

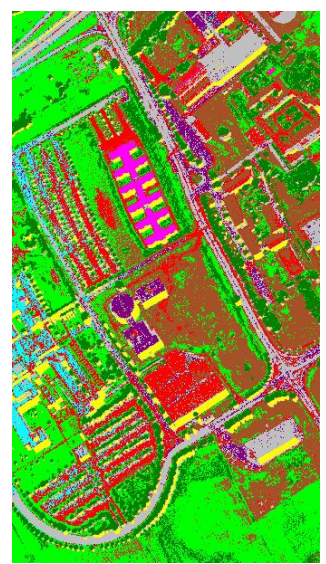

(b)

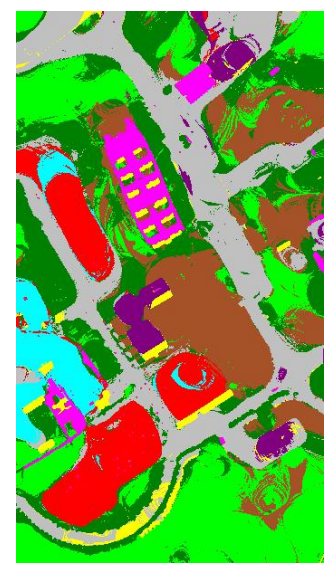

(c)

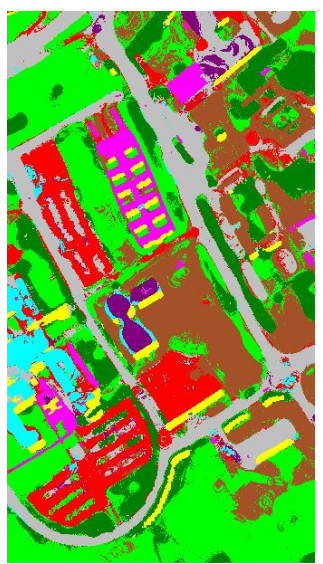

(d)

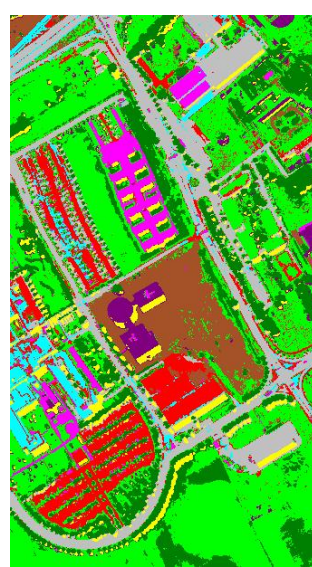

(e)

Fig. 6. Classification maps of University of Pavia ROSIS image (20 samples per class). (a) SpeRoF, OA $=77.37 \%$. (b) SSRoF, OA $=80.14 \%$. (c) SSRoF $\mathrm{OA}=88.55 \%$. (d) $\mathrm{SSRoF}_{M S}, \mathrm{OA}=92.14 \%$. (e) RS-EMAPs, $\mathrm{OA}=90.33 \%$.

TABLE VI

INDIAN PINES AVIRIS (10 SAMPLES PER CLASS): SENSITIVITY TO THE CHANGE OF $M$.

\begin{tabular}{c|c|c|c|c}
\hline \hline $\mathrm{M}$ & 10 & 25 & 55 & 110 \\
\hline SSRoF & 54.64 & 56.86 & 58.82 & 60.05 \\
SSRoF $_{\text {opt }}$ & 79.11 & 79.21 & 79.65 & 79.67 \\
SSRoF $_{M S}$ & 81.69 & 81.97 & 81.29 & 82.55 \\
\hline \hline
\end{tabular}

the classification performances of $\mathrm{SSRoF}_{\text {opt }}$ are significantly improved when compared to SSRoF. The OAs of SSRoF with different scales are shown in Fig. 4 . Different scales achieve various performances for two hyperspectral images. For instance, SSRoF with a neighborhood size of $11 \times 11$ delivers the best performance for Indian Pines AVIRIS image, whereas SSRoF with a neighborhood size of $15 \times 15$ gets the highest OA for University of Pavia Image. To take all the advantages of different scales, we proposed to combine their classification results using a majority vote rule. As we expected, $\mathrm{SSRoF}_{M S}$ performs the best performance. Furthermore, according to the Mcneamar statistic test 1 presented in Tables III and IV], our proposed $\mathrm{SSRoF}_{M S}$ is superior and comparable to the spectral-spatial classifiers. For Indian Pines AVIRIS image, when the small sizes of training set (e.g., 10, $15, . ., 30$ samples per class) is adopted, there is no difference between our proposed method and RS-EMAPs classifier. In other cases, our proposed method is significantly better than other classifiers. Figs. 5 and 6 shows the obtained classification maps provided by different classification methods for the two images. As shown in Figs. 5 and 6 the classification map produced by $\mathrm{SSRoF}_{M S}$ is more accurate than those generated by other algorithms.

In the second experiment, besides OA, the percentage average overall accuracies of the individual DT classifier, "AOA (\%)," and the coincident failure diversity (CFD) [34] are added

\footnotetext{
${ }^{1}$ McNemar's test is computed by $Z=\frac{f_{12}-f_{21}}{\sqrt{f_{12}+f_{21}}} \cdot f_{12}$ represents the number of samples classified correctly by classifier 1 and incorrectly by classifier 2 . The difference between classifiers 1 and 2 is to be statistically significant if $|Z|>1.96$ with $\alpha=0.05$ using two-tail test. $Z>0$ indicates that classifier 1 is more accurate than classifier 2 .
}

TABLE VII

UNIVERSITY OF PAVIA ROSIS (10 SAMPLES PER CLASS): SENSITIVITY TO THE CHANGE OF $M$.

\begin{tabular}{c|c|c|c}
\hline \hline $\mathrm{M}$ & 10 & 25 & 50 \\
\hline SSRoF & 73.05 & 68.81 & 65.77 \\
SSRoF $_{o p t}$ & 82.51 & 82.56 & 81.49 \\
SSRoF $_{M S}$ & 86.80 & 86.46 & 84.21 \\
\hline \hline
\end{tabular}

TABLE VIII

INDIAN PINES AVIRIS (10 SAMPLES PER CLASS): SENSITIVITY TO THE CHANGE OF $\phi$.

\begin{tabular}{c|c|c|c|c|c}
\hline \hline$\phi$ & 0.1 & 0.3 & 0.5 & 0.7 & 0.9 \\
\hline SSRoF & 59.64 & 59.81 & 60.05 & 60.04 & 60.17 \\
SSRoF $_{\text {opt }}$ & 78.99 & 79.65 & 79.67 & 79.14 & 79.54 \\
SSRoF $_{M S}$ & 81.99 & 82.65 & 82.55 & 82.14 & 82.38 \\
\hline \hline
\end{tabular}

TABLE IX

UNIVERSITY OF PAVIA ROSIS (10 SAMPLES PER CLASS): SENSITIVITY TO THE CHANGE OF $\phi$.

\begin{tabular}{c|c|c|c|c|c}
\hline \hline$\phi$ & 0.1 & 0.3 & 0.5 & 0.7 & 0.9 \\
\hline SSRoF & 72.07 & 72.46 & 73.05 & 72.49 & 73.45 \\
SSRoF $_{o p t}$ & 81.96 & 81.78 & 82.51 & 82.54 & 82.64 \\
SSRoF $_{M S}$ & 86.41 & 86.52 & 86.80 & 86.74 & 86.81 \\
\hline \hline
\end{tabular}

to analyze SpeRoF, SpaRoF, and SSRoF. A stronger diversity is represented by a higher value of $C F D$. As shown in Table $\mathrm{V}$ higher values of AOAs and diversities achieved by SSRoF are greater than those obtained from SpeRoF and SpaRoF, leading to better classification performance.

In the third experiment, we focus on the analysis of the sensitivity of two parameters, $M$, which represents the number of features in a subset, and $\phi$, which is the control parameter between spectral and spatial information for the construction of SSRoF. The results of such analysis are shown in Tables. VI, VII VIII, and IX From Table. VI, as $M$ increase, SSRoF tends to have better performance for the AVIRIS image. However, the situation in ROSIS image is exactly reversed (Table. VII]. The results are consistent with our previous studies [16]. 
In conclusion, $M$ has a significant influence on the classification result. This parameter is application dependent. However, when the WMF is introduced into SSRoF, the classification performances of $\mathrm{SSRoF}_{\text {opt }}$ and $\mathrm{SSRoF}_{M S}$ become very stable. Moreover, from Tables. VIII] and IX, larger values of $\phi$ could yield the better classification results. However, the difference is quite small (less than 0.7 percentage points) and not significant according to the McNemar's test. Therefore, we can conclude that the proposed method is not sensitive to $\phi$.

In our previous studies, we have developed rotation random forest (RoRF) via kernel PCA (KPCA) [35], and the combination of rotation forest and multi-scale segmentation [36] for the classification of hyperspectral images. The former method used KPCA instead of PCA in the construction of RoRF. Although it can achieve better performance than RoF and RoRF with spectral information, the computational time is much longer than the ones of RoF and RoRF. The latter method applied RoF to classify the objects extracted from the multi-scale segmentation techniques (i.e., eCognition software) and then combined the results to generate the final result. In the eCognition, we should carefully configure the settings of segmentations, such as the scales, spectral and shape heterogeneity. Compared to the two mentioned methods, our proposed method is simple but effective. The computational time is short than RoRF via KPCA. For the parameter selection, we only consider the scales.

\section{CONClusions AND PERSPECTIVES}

In this work, a spectral and spatial Rotation Forest (SSRoF) is proposed for hyperspectral image classification. We aim at improving RoF in two aspects: 1) using multi-scale WMF and 2) introducing spectral-spatial data transformation into RoF. The main conclusion can be drawn as follows:

- By introducing spectral-spatial data transformation, SSRoF is superior to SpeRoF and SpaRoF.

- With the help of multi-scale WMF, the proposed $\mathrm{SSRoF}_{M S}$ gains the highest classification results.

- The proposed algorithm is very competitive with other state-of-the-art spectral-spatial classifiers.

The future studies will include, but will not be limited to, the following topics.

- We will include more base classifiers into our SSRoF framework.

- We will adopt our proposed method into other datasets, such as LiDAR, or other applications, such as change detection.

\section{ACKNOWLEDGMENT}

The authors would like to thank Prof D. Landgrebe from Purdue University and Prof P. Gamba from University of Pavia for providing the hyperspectral datasets.

\section{REFERENCES}

[1] C. I. Chang, Hyperspectral Imaging: Techniques for Spectral Detection and Classification. Plenum Publishing Co., 2003.

[2] C. I. Chang, Hyperspectral Data Exploitation: Theory and Applications. Wiley-Interscience, Hoboken, NJ, 2007.
[3] G. Hughes, "On the mean accuracy of statistical pattern recognizers," IEEE Trans. Infor. Theory, vol. 14, no. 1, pp. 55-63, 1968.

[4] D. Lu and Q. Weng, "A survey of image classification methods and techniques for improving classification performance," International Journal of Remote Sensing, vol. 28, no. 5, pp. 823-870, 2007.

[5] J. A. Benediktsson, J. Chanussot, and M. Fauvel, "Multiple classifier systems in remote sensing: from basics to recent developments," in Proceedings of the 7th International Workshop on Multiple Classifier Systems, Prague, Czech Republic, May 23-25, 2007, pp. 501-512.

[6] Y. Tarabalka, J. A. Benediktsson, and J. Chanussot, "Spectral-spatial classification of hyperspectral imagery based on partitional clustering techniques," IEEE Trans. Geosci. Remote Sens., vol. 47, no. 8, pp. 29732987, 2009.

[7] A. J. Brown, "Spectral curve fitting for automatic hyperspectral data analysis," IEEE Trans. Geosci. Remote Sens., vol. 44, no. 6, pp. 16011608, June 2006.

[8] A. J. Brown, B. Sutter, and S. Dunagan, "The marte imaging spectrometer experiment: Design and analysis," Astrobiology, vol. 8, no. 5, pp. 1001-1011, 2008.

[9] F. Melgani and L. Bruzzone, "Classification of hyperspectral remote sensing images with support vector machines," IEEE Trans. Geosci. Remote Sens., vol. 42, no. 8, pp. 1778-1790, 2004.

[10] G. Camps-Valls and L. Bruzzone, "Kernel-based methods for hyperspectral image classification," IEEE Trans. Geosci. Remote Sens., vol. 43, no. 6, pp. 1351-1362, 2005.

[11] P. Du, J. Xia, W. Zhang, K. Tan, Y. Liu, and S. Liu, "Multiple classifier system for remote sensing image classification: A review," Sensors, vol. 12, no. 4, pp. 4764-4792, 2012.

[12] L. I. Kuncheva, Combining Pattern Classifiers: Methods and Algorithms. Wiley-Interscience, 2004.

[13] L. Breiman, "Random forest," Machine Learn., vol. 45, no. 1, pp. 5-32, 2001.

[14] J. J. Rodriguez and L. I. Kuncheva, "Rotation forest: A new classifier ensemble method." IEEE Trans. Pattern Anal. Mach. Intell, vol. 28 , no. 10, pp. 1619-1630, 2006.

[15] J. Xia, J. Chanussot, P. Du, and X. He, "Rotation-Based Ensemble Classifiers for High-Dimensional Data," in Fusion in Computer Vision, B. Ionescu, J. Benois-Pineau, T. Piatrik, and G. Quénot, Eds. Springer, 2014 , pp. $135-160$

[16] J. Xia, J. Chanussot, P. Du, and X. He, "Spectral-spatial classification for hyperspectral data using rotation forests with local feature extraction and markov random fields," IEEE Trans. Geosci. Remote Sens., vol. 53, no. 5, pp. 2532-2546, 2015.

[17] J. Xia, P. Du, X. He, and J. Chanussot, "Hyperspectral remote sensing image classification based on rotation forest," IEEE Geosci. Remote Sens. Lett., vol. 11, no. 1, pp. 239 - 243, 2014.

[18] J. Xia, J. Chanussot, P. Du, and X. He, "Rotation-based support vector machines in classification of hyperspectral data with limited training samples," IEEE Trans. Geosci. Remote Sens., in press.

[19] J. Xia, M. Dalla Mura, J. Chanussot, P. Du, and X. He, "Random subspace ensembles for hyperspectral image classification with extended morphological attribute profiles," IEEE Trans. Geosci. Remote Sens., vol. 53, no. 9, pp. 4768-4786, 2015.

[20] T. Kavzoglu, I. Colkesen, and T. Yomralioglu, "Object-based classification with rotation forest ensemble learning algorithm using very-highresolution worldview-2 image," Remote Sensing Letters, vol. 6, no. 11, pp. 834-843, 2015.

[21] P. Du, A. Samat, B. Waske, S. Liu, and Z. Li, "Random forest and rotation forest for fully polarized SAR image classification using polarimetric and spatial features," ISPRS J. Photogramm Remote. Sens., vol. 105 , pp. $38-53,2015$.

[22] M. Sugiyama, "Dimensionality reduction of multimodal labeled data by local Fisher discriminant analysis," J. Mach. Learn. Res., vol. 27, no. 8 , pp. 1021-1064, 2007.

[23] Y. Zhou, J. Peng, and C. Chen, "Dimension reduction using spatial and spectral regularized local discriminant embedding for hyperspectral image classification," IEEE Trans. Geosci. Remote Sens., vol. 53, no. 2, pp. 1082-1095, 2015

[24] Y. Tarabalka, J. Chanussot, and J. A. Benediktsson, "Segmentation and classification of hyperspectral images using watershed transformation," Pattern Recogn., vol. 43, no. 7, pp. 2367-2379, 2010.

[25] C. Chen, W. Li, H. Su, and K. Liu, "Spectral-spatial classification of hyperspectral image based on kernel extreme learning machine," Remote Sensing, vol. 6, no. 6, p. 5795, 2014.

[26] Y. Chen, X. Zhao, and X. Jia, "Spectral-spatial classification of hyperspectral data based on deep belief network," IEEE Journal of Selected 
Topics in Applied Earth Observations and Remote Sensing, vol. 8, no. 6, pp. 2381-2392, June 2015.

[27] K. He, J. Sun, and X. Tang, "Guided image filtering," IEEE Trans. on Pattern Anal. Machine Intell., vol. 35, no. 6, pp. 1397-1409, June 2013.

[28] X. Kang, S. Li, and J. A. Benediktsson, "Spectral-spatial hyperspectral image classification with edge-preserving filtering," IEEE Trans. Geosci. Remote Sens., vol. 52, no. 5, pp. 2666-2677, 2014.

[29] X. Kang, S. Li, and J. A. Benediktsson, "Feature extraction of hyperspectral images with image fusion and recursive filtering," IEEE Trans. Geosci. Remote Sens., vol. 52, no. 6, pp. 3742-3752, 2014.

[30] J. Xia, L. Bombrun, T. Adal, Y. Berthoumieu, and C. Germain, "Spectral-spatial classification of hyperspectral images using ICA and edge-preserving filter via an ensemble strategy," IEEE Trans. Geosci. Remote Sens., vol. 54, no. 8, pp. 4971-4982, Aug 2016.

[31] Q. Zhang, L. Xu, and J. Jia, "100+ times faster weighted median filter (WMF)," in IEEE Conference on Computer Vision and Pattern Recognition (CVPR), Columbus, Ohio, USA, June 24-27, 2014.

[32] J. Li, J. M. Bioucas-Dias, and A. Plaza, "Hyperspectral image segmentation using a new Bayesian approach with active learning," IEEE Trans. Geosci. Remote Sens., vol. 49, no. 12, pp. 3947-3960, 2011.

[33] G. Camps-Valls, L. Gomez-Chova, J. Munoz-Mari, J. Vila-Frances, and J. Calpe-Maravilla, "Composite kernels for hyperspectral image classification," IEEE Geoscience and Remote Sensing Letters, vol. 3, no. 1, pp. 93-97, Jan 2006.

[34] L. I. Kuncheva and C. J. Whitaker, "Measures of diversity in classifier ensembles and their relationship with the ensemble accuracy," Mach Learn., vol. 51, no. 2, pp. 181-207, 2003.

[35] J. Xia, N. Falco, J. A. Benediktsson, P. Du, and J. Chanussot, "Hyperspectral image classification with Rotation Random Forest via KPCA," IEEE J. Sel. Topics Appl. Earth Observ. Remote Sens., vol. 10, no. 4, pp. 1601-1609, April 2017.

[36] J. Chen, J. Xia, P. Du, and J. Chanussot, "Combining rotation forest and multiscale segmentation for the classification of hyperspectral data," IEEE J. Sel. Topics Appl. Earth Observ. Remote Sens., vol. 9, no. 9, pp. 4060-4072, Sept 2016.

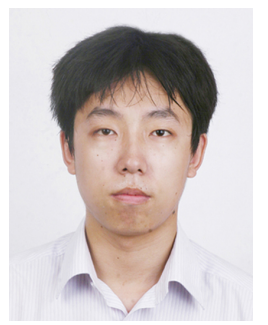

Junshi Xia (S'11-M'16) received the B.S. degree in geographic information systems and the Ph.D. degree in photogrammetry and remote sensing from the China University of Mining and Technology, Xuzhou, China, in 2008 and 2013, respectively. He also received the Ph.D. degree in image processing with the Grenoble Images Speech Signals and Automatics Laboratory, Grenoble Institute of Technology, Grenoble, France, in 2014. From December 2014 to April 2015, he was a Visiting Scientist with the Department of Geographic Information Sciences, Nanjing University, Nanjing, China. From May 2015 to April 2016, he was a Postdoctoral Research Fellow with the University of Bordeaux, Talence, France. From May 2016, he is the JSPS Postdoctoral Oversea Research Fellow with the University of Tokyo, Tokyo, Japan. His research interests include multiple classifier system in remote sensing, hyperspectral remote sensing image processing, and urban remote sensing.

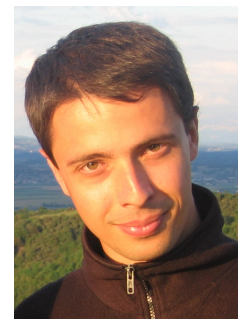

Lionel Bombrun was born in Tournon, France, in 1982. He received the M.S. and Ph.D. degrees in signal, image, speech, and telecommunications from the Grenoble National Polytechnic Institute, Grenoble, France, in 2005 and 2008, respectively. In 2008, he was a Teaching Assistant with Phelma, Grenoble. From October 2009 to September 2010, he was a Postdoctoral Fellow with the French National Council for Scientific Research (CNRS) between the Grenoble Image Speech Signal Automatics Laboratory and SONDRA, Gif-sur-Yvette, France. From October 2010 to August 2011, he was a Postdoctoral Fellow with IMS Bordeaux (UMR 5218). He is currently an Associate Professor with Bordeaux Sciences Agro and a member of the IMS Lab. His research interests include image processing, stochastic modeling, and texture analysis

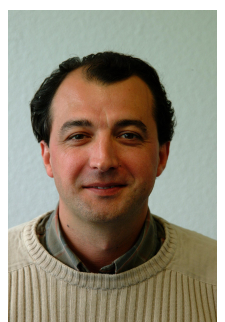

Yannick Berthoumieu (M'09) received the Ph.D. degree in signal processing from the University of Bordeaux I, Talence, France, in 1996. In 1998, he joined the Ecole Nationale Supérieure d'Electronique, Informatique et Radiocommunications de Bordeaux, Talence, as an Associate Professor. He is currently a Full Professor with the Department of Telecommunications, Bordeaux National Institute of Technology. Since 2003, he has been with the Signal and Image Processing Group, IMS Lab, University of Bordeaux. His major research interests include multidimensional signal analysis, stochastic modeling, texture analysis, and video processing.

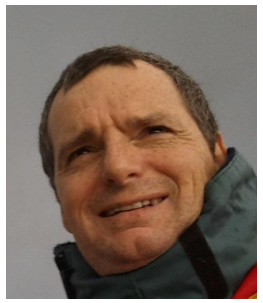

Christian Germain received the M.Sc. degree in automatic control and signal processing and the $\mathrm{Ph} . \mathrm{D}$. degree from the University of Bordeaux, Talence, France, in 1993 and 1997, respectively. He has taught computer science and image processing since 1984, and he is currently a Professor with Bordeaux Sciences Agro. He is carrying out research in the Signal and Image Group, IMS Lab (CNRS, Bordeaux University, IPB). His research interests include orientation estimation and texture analysis and synthesis in two and three dimensions. Applications concern material characterization, artificial vision for agriculture, and highresolution remote sensing.

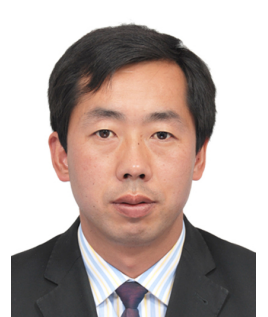

Peijun Du (M07-SM12) received the Ph.D. degree in geomatics engineering from China University of Mining and Technology, Xuzhou, China, in 2001. He was a Postdoctoral Fellow with Shanghai Jiao Tong University, Shanghai, China, from February 2002 to March 2004, and was a Senior Visiting Scholar at the University of Nottingham, Nottingham, U.K., and the Grenoble Images Speech Signals and Automatics Laboratory (GIPSA-lab), Grenoble Institute of Technology, Grenoble, France. He is a Professor with the Remote Sensing, Department of Geographic Information Sciences, Nanjing University, Nanjing, China, and the Deputy Director with the Key Laboratory for Satellite Mapping Technology and Applications of National Administration of Surveying, Mapping, and Geoinformation (NASG), Beijing, China. His research interests include remote sensing image processing and pattern recognition, hyperspectral remote sensing, and applications of geospatial information technologies.

Dr. Du has been the Associate Editor of the IEEE GEOSCIENCE AND REMOTE SENSING LETTERS (GRSL) since 2009. He was the Guest Editor of three special issues IEEE JOURNAL OF SELECTED TOPICS IN APPLIED EARTH OBSERVATION AND REMOTE SENSING (JSTARS). $\mathrm{He}$ also served as the Co-Chair of the Technical Committee of URBAN 2009, EORSA 2014, and IAPR-PRRS 2012, the Co-Chair of the Local Organizing Committee of JURSE 2009, WHISPERS 2012, and EORSA 2012, and the member of Scientific Committee or Technical Committee of other international conferences, e.g., Spatial Accuracy 2008, ACRS 2009, WHISPERS (20102016), URBAN (2011, 2013, and 2015), MultiTemp (2011, 2013, and 2015), ISDIF 2011, and SPIE European Conference on Image and Signal Processing for Remote Sensing (2012-2016). 